\title{
RESEÑAS
}




\section{EVOLUCION DE LA POLITICA EDUCATIVA DURANTE EL SIGLO XX PRIMERA PARTE 1900-1957}

Molano, Alfredo; Vera, César*

Acaba de aparecer el primer volumen (1900-1957) de este importante estudio que trata de llenar un vacío grande en la historiografía nacional que, en este campo particular, ya no podía sostenerse con los análisis un tanto aproximativos y escasamente documentados de Ivon Lebot. Se espera, más adelante, con el segundo volumen, cubrir el período restante (la Junta Militar y los cuatro gobiernos del Frente Nacional) para así sacar a luz las líneas más sobresalientes de la Política Educativa en nuestra historia contemporánea.

Dos juiciosos investigadores, con trabajos anteriores en este campo, rastreando una importante documentación, han querido de esta manera contestar a las preguntas centrales de un tema que por su objeto y significación puede servir de índice de lectura a una de las grandes líneas de desarrollo nacional. Resulta por ello importante intentar una breve reseña, con todo lo problemático que esto resulta, sobre las principales conclusiones del trabajo en curso y poder, de esta manera, señalar algunas observaciones tanto teóricas como metodológicas y documentales, intentando un corto y arriesgado balance que vaya más allá del "Alabado, Alabado" con que las pésimas costumbres intelectuales de los "reseñistas" saludan la aparición de cualquier texto. Con esta mira me detendré en los dos primeros capítulos, pues creo que ahí se juega lo esencial: (El Régimen Conservador: 1900 - 1930 y La República Liberal: 1930-1946) que, como se ve por sus propios títulos y fechas, dan cuenta de una periodización ya tradicional de lo Político aceptada como una evidencia para el campo de los fenómenos educativos.

Con algunos acentos y matices el estudio en consideración desenvuelve sus conclusiones en el marco ya normal" y conocido de otros análisis que sobre el mismo campo o campos relacionados han presentado otros investigadores del período. Resumo muy sucintamente:

1) "...el régimen conservador fue temerariamente refractario a todo tipo de reformas educativas a pesar de que los cambios económicos y sociales... eran inobjetables". ( $p$. 39) "Los continuos y vigorosos cambios sociales del período contrastaron con la rigidez de la política educativa del gobierno de (Pedro Nel) Ospina". O, "De 1904 a 1920 la política educativa se mantiene invariable" (p. 22), y esto porque "Las transformaciones (económico-sociales, r.s.) sus ecos y sus signos, no lograron dejar huella en el sistema educativo". En estos años de hegemonía conservadora, en opinión de los autores, no se pueden detectar sino cambios menores. Un ejemplo; "La Ley 28 de 1925, que decretó las fiestas nacionales de la bandera y de la madre y la Ley 12 de 1926 sobre la enseñanza de la higiene..."

2) Sin embargo, parece que para los autores todo cambia con el advenimiento de la República Liberal, siguiendo en su análisis la ruta trazada por el libro de Álvaro Tirado Mejía sobre el primer gobierno de López Pumarejo, libro en que tal tendencia de análisis ya había sido llevada a su punto más elevado. "La indiferencia por la

\footnotetext{
${ }^{*}$ Universidad Pedagógica Nacional, centro de Investigaciones, Bogotá, 1984, 188 p.
} 
problemática educativa (indiferencia que nunca ha existido en más de siglo y medio de vida independiente, r.s.) comienza a ceder paso a una conciencia creciente sobre (sus) funciones..." (p. 112). Desde muy temprano, "con las medidas adoptadas por el gobierno de Olaya Herrera la política educativa comienza a alejarse de la tradición conservadora y a introducir una nueva orientación..." para de esta manera ir "adecuando el sistema educativo a la estructura productiva engendrada por la industrialización" (p. 112). El aspecto más avanzado y profundo de esta nueva orientación estaría dado por el gobierno de López Pumarejo, pues "La primera administración de López transformó sustancialmente el sistema educativo vigente desde principios de siglo, (hecho) reconocido por todos los investigadores que se han ocupado del tema". Y no sólo por los distintos investigadores del período sino por el propio López quien fue propiamente quien inició la versión que nunca ha dejado de circular. Como lo señalan los propios autores: "López mismo era consciente de la trascendencia del proceso en que se empeñó", fieles a la imagen que un período y sus criaturas dieron como interpretación de sus tareas. Hasta ahí, con alguna injusticia, las principales conclusiones: El país comenzó a principios de siglo un proceso de industrialización que no fue seguido de inmediato por las tareas de adecuación del viejo sistema educativo a la nueva estructura productiva, tarea que llevó adelante, aunque no a su término, la República Liberal.

Quisiera por mi parte tratar de relativizar este análisis, señalar algunos de sus inconvenientes y mostrar las posibilidades de su profundización, no en el sentido simple de extender y reiterar una interpretación (que de por si puede ser siempre prolongada) sino mas bien en un intento de indicar cómo, a mi juicio, tal interpretación puede hacerse más certera, más eficaz, más ajustada a un esfuerzo por desconstruir las visiones tradicionales de la historia y sus períodos en nuestro medio.

El análisis que presentan en su libro Molano y Vera está sustentado en un sólo principio que, de manera desafortunada, aparece indiscutido a lo largo del texto. Quien mire, aún sin mayor detenimiento, el "orden de exposición" del proceso que se nos va a relatar, fácilmente lo encontrará; de la economía a la educación. "Se trataba de explicar el sistema educativo (...) por la estructura social y económica que lo enmarcaba..." (p. 5) Ese principio, que fue planteado desde el inicio de la empresa investigativa y que permaneció intacto a lo largo de sus diversos cambios, supone una particular concepción de la causalidad, y, por tanto, de las relaciones entre "lo determinante y lo determinado" en el marco de una estructura social.

Empecemos por esto último, aunque se trate de problemas estrechamente relacionados. Cuando se mantiene como un rígido principio explicativo, $-\mathrm{y}$ no como una hipótesis de trabajo en permanente examen-, la posición privilegiada de la "economía" frente a los otros procesos sociales, esos "otros" siempre terminan apareciendo como "derivados", como "subsidiarios", pues la concepción que sigue presente en el centro del análisis resulta ser entonces la del "reflejo", regida por la pareja adecuacióninadecuación. Y no simplemente porque no existan tales fenómenos de "adecuacióninadecuación" sino porque la actividad investigativa no se dedica a constatarlos (actitud empirista) sino que busca explicarlos, mostrando de manera concreta cómo en una sociedad sus diversos elementos no se modifican y cambian bajo la misma temporalidad, bajo el mismo ritmo; cómo cada elemento del proceso (y de ahí la "adecuacióninadecuación") obedece a una genealogía propia y diferencial. En general, el desconocimiento de este hecho lleva casi siempre, a la vista de unos resultados legales, 
en el análisis del fenómeno educativo del período, a una falsa valoración de la actividad de los partidos políticos, valoración regida en silencio por la pareja reacción-progreso. Y no porque haya que abandonar una conquista congénita del saber social contemporáneo que asigna, como principio teórico, un peso específico a las "estructuras materiales" de una sociedad en la determinación de su forma, sino porque tal principio teórico debe transformarse, metodológicamente para cada investigación, en un supuesto sometido a interrogación.

En cuanto a la causalidad propiamente dicha habría que seguir reclamando una concepción más dinámica y flexible que permita inscribir el conjunto de elementos dispersos que conforman un proceso en un juego múltiple de relaciones, sustituyendo la visión aristotélico-tomista de la causa-efecto por una concepción más móvil; por ejemplo (y pienso en una como la que aparece en el siempre citado Capítulo XXIV del Tomo 1 del Capital), una que nos plantee para todo proceso complejo la existencia de un conjunto de determinaciones que vienen a ser "condición y resultado del proceso". Y no porque haya que temer a la determinación de relaciones precisas de causalidad sumándose a la concepción ecléctica de los "diversos factores de peso imponderable" sino porque cada uno de ellos debe ser siempre sometido a un examen riguroso que permita conocer para cada proceso su forma de funcionamiento.

Sea el caso de la llamada industrialización. La industrialización, como proceso de implantación de la relación capitalista de dominación, y de sus resultados a diverso nivel, no es un proceso de forma universal o el desenvolvimiento de leyes universales que se "aplican" a "casos" particulares. Es un proceso histórico-concreto de múltiples formas diferenciales que deben ser estudiadas en su singularidad. Y nada permite suponer la existencia de una relación fija entre tal proceso y los fenómenos educativos que se le asocian, una relación de determinante y determinado. Se trata de series que se elaboran en distintos espacios y niveles, bajo distintas relaciones de fuerza, que no encuentran las mismas resistencias, que no obedecen a la misma "duración" y que pueden elaborarse de manera relacionada o no, de manera simultánea o no.

Tratándose de la relación entre proceso industrial y proceso educativo, una relación que puede ( $y$ debe) ser estudiada ( $y$ de hecho lo ha sido, como lo muestra el texto que comenzamos) hay que tener en cuenta que la industrialización da lugar a cambios precisos en la "población", pero que también los supone (más allá de la simple liberación de la fuerza de trabajo de formas serviles o artesanales): un nivel de saber y de calificación técnica para el trabajo, nuevas relaciones con el espacio, con el tiempo y con el cuerpo, y un foco de esa transformación está dado por el sistema educativo que resulta ser así condición y resultado del proceso. Sin introducir en el análisis el concepto de "población" y de "cuerpo" (entendidos aquí como un objeto de las relaciones y estrategias de poder) habrá demasiadas cosas que por mucho tiempo seguirán siendo oscuras.

En nuestra historia contemporánea, la que más de cerca nos toca y quema, existen demasiadas evidencias documentales que muestran que desde principios de siglo los objetos población-educación-cuerpo fueron un blanco central de los poderes y un campo de acción privilegiado de la política educativa del Estado en formación (sobre todo en el período conservador, en contra de lo que habitualmente creen y escriben los modernos historiadores). Los grandes estudios sobre el alcoholismo y la chicha de finales del siglo pasado y principios del presente; la crucial discusión sobre el futuro de la raza iniciada en pésimos términos - por Armando Solano: toda una intelectualidad ácremente discutiendo sobre un pueblo que había sido exánime de su más larga guerra; la formalización de un instrumento central de trabajo sobre el cuerpo como es el servicio 
militar; la lucha - aún no terminada-- por calzar un pueblo, bajo el lema de "Calzarse es civilizarse"; la entrada en la Escuela, al lado del cura y del maestro, del médico, y el privilegio de la higiene, que más tarde permitiría a un joven ministro de Educación decir: "En Colombia el problema no es educar sino higienizar". Pero en el estudio de la historia de la educación y de las políticas educativas la relación población-educación-cuerpo no puede ser trabajada en forma correcta si, contentándonos con los cambios en los contenidos y las "materias", y declarando que la celebración del día de la madre y la implantación de la higiene son poca cosa, seguimos desatendiendo el análisis de las "técnicas pedagógicas" como instrumentos inscritos en el dispositivo general del Orden.

Una observación más me gustaría señalar: buena parte de la "originalidad educativa" asignada a la República Liberal depende solamente de un espejismo. Si en el análisis del problema seguimos contentándonos con el nivel legal y nuestra documentación sigue siendo la legislación y las memorias casi exclusivamente, si no abrimos paso a nuevas series documentales producidas en espacios y micro-espacios distintos de la actividad estatal, seguiremos recayendo en la trampa de pensar que la Ley crea y produce, y desconociendo que actúa sobre y reglamenta situaciones que las prácticas concretas han venido generando. En el caso analizado pudiera resultar que el privilegio de la información legal, oficial y estatal impidiera ver los diferentes lugares en que fueron creándose en los años anteriores los elementos de la política educativa que luego el liberalismo formalizó, elementos que no son simplemente del orden de las condiciones de posibilidad (la industrialización) sino del orden de la genealogía de cada elemento concreto que aparece en la orientación educativa del período, pues si bien todos sabemos que una legislación por sí misma no implica un cambio, todos debemos recordar también que ella no se genera a nivel de la Ley ni de las simples condiciones de posibilidad, que pueden ser un "contexto" de gran importancia, pero no el único elemento que explica la existencia y vigencia de una política educativa determinada.

Renán Silva 